\title{
Stable inheritance of telomere chromatin structure and function in the absence of telomeric repeats
}

\author{
Mahito Sadaie, ${ }^{1}$ Taku Naito, ${ }^{1}$ and Fuyuki Ishikawa ${ }^{1,2,3}$ \\ ${ }^{1}$ Laboratory of Molecular and Cellular Assembly, Department of Biological Information, Graduate School of Bioscience \\ and Biotechnology, Tokyo Institute of Technology, Yokohama 226-8501, Japan; ${ }^{2}$ Laboratory of Cell Cycle Regulation, \\ Department of Gene Mechanisms, Graduate School of Biostudies, Kyoto University, Kyoto 606-8502, Japan
}

\begin{abstract}
It is generally believed that telomeric repeats are a necessary and sufficient cis-element for telomere function. Here we show that telomere structure and meiotic function are stably inherited in fission yeast circular chromosomes that have lost all telomeric repeats. We found that the telomeric repeat binding protein, Taz1, and the heterochromatin protein, Swi6, remain associated with subtelomeres in the absence of telomeric repeats. We also found that the fusion point of circular chromosomes that lack telomeric repeats associates with SPB (the yeast counterpart of the centrosome) in the premeiotic horsetail stage, similarly to wild-type telomeres. However, a taz1 ${ }^{+}$deletion/reintroduction experiment revealed that the maintenance of Taz1 binding and premeiotic function is achieved via different strategies. Taz1 is recruited to subtelomeres by an autonomous element present in subtelomeric DNA, thus in a genetic mechanism. In contrast, the premeiotic subtelomere-SPB association is maintained in an epigenetic manner. These results shed light on the previously unrecognized role played by the subtelomere and underscore the robust nature of the functional telomere complex that is maintained by both genetic and epigenetic mechanisms. Furthermore, we suggest that the establishment and the maintenance of the functional telomere complex are mechanistically distinguishable.
\end{abstract}

[Keywords: Telomere; subtelomere; epigenetic inheritance; circular chromosome; meiosis; Taz1]

Supplemental material is available at http://www.genesdev.org.

Received May 12, 2003; revised version accepted July 21, 2003.

The transmission of chromosomes from one cell to daughter cells lies at the heart of the continuity of life. Three chromosomal domains-centromeres, telomeres, and replication origins-are necessary for this process. Although their structures and functions are highly faithful and rigid in general, they show surprisingly flexible and adaptable behavior on some occasions. An example of such can be seen in centromeres. Centromeres are frequently inactivated in dicentric chromosomes and occasionally established de novo at noncentromeric DNA in acentric chromosomes (neocentromere; Karpen and Allshire 1997; Sullivan et al. 2001). In most cases, neocentromere formation and centromere inactivation are explained by an epigenetic mechanism. In this context, once a specific chromatin structure conferring centromeric function is established, it is inherited by replication of the pre-existing chromatin during cell division in a self-templating manner. A similar epigenetic inheri-

${ }^{3}$ Corresponding author.

E-MAIL fishikaw@lif.kyoto-u.ac.jp; FAX 81-75-753-4197.

Article published online ahead of print. Article and publication date are at http://www.genesdev.org/cgi/doi/10.1101/gad.1112103. tance of chromatin has been well documented in yeast heterochromatin at the centromere or mating-type locus (Allshire et al. 1994; Grewal and Klar 1996).

Although telomeres share features characteristic of heterochromatin with centromeres and the mating-type locus, their epigenetic nature is less understood. Telomeres consist of telomeric repeats, tandem arrays of short G/C-rich sequences, and associated proteins. Telomeres have numerous biological functions. First, they protect the chromosomal ends from enzymatic degradation, DNA end-to-end fusion, and from being recognized by the DNA damage checkpoint machinery. Second, they counteract the end-replication problem by recruiting telomerase, a telomere-specific reverse transcriptase. Telomeres also play an important role in meiosis, because all telomeres in one cell become clustered in a specific stage of meiosis (Scherthan 2001), thereby promoting homologous chromosome pairing (Cooper 2000).

The ectopic introduction of telomeric repeats nucleates and establishes telomeres de novo (Gottschling et al. 1990; Barnett et al. 1993; Nimmo et al. 1994). "Chromosome healing" by the de novo addition of telomeric repeats to the broken ends of chromosomes is frequently 
observed in cancer cells (Varley et al. 2000 and references therein). On the other hand, when telomeric repeats are inducibly eliminated from an extra nonessential chromosome in yeast, the chromosome is lost from the cells (Sandell and Zakian 1993). Therefore, telomeric repeats are considered to be a necessary and sufficient cis-element for telomere function. However, it is not known whether telomere function exhibits any adaptable behavior under selective conditions such as those in cancer cell populations, which frequently show extremely short telomeres.

Centromeric to the telomeric repeats is the subtelomeric region, which is characterized in many organisms by a relatively low gene density and the presence of lowcopy repetitive sequences (Mefford and Trask 2002). As the telomeres newly formed by the chromosome healing mechanism do not contain the subtelomeric region yet are functional, it is generally believed that the subtelomeric region is dispensable for most, if not all, telomere functions.

Previously, we and others reported that fission yeast survives in the absence of telomeric repeats by rendering all three chromosomes circular (Naito et al. 1998; Nakamura et al. 1998). During the investigation of the meiotic behavior of circular chromosomes, we found a hitherto undescribed robust nature of telomeres: At least part of the telomere-associated functions, specifically meiotic telomere clustering, and chromatin structures were inherited stably in the absence of telomeric repeats. These results provide a new avenue in our understanding of the robust nature of telomere function.

\section{Results}

In this study, we examined circular chromosomes formed in $\Delta$ trt1, a null mutant of the telomerase catalytic subunit. Eight independent Schizosaccharomyces pombe $\Delta$ trt1 clones harboring circular chromosomes in the $h^{90}$ background were isolated (hereafter called derivative clones). Different clones were produced from independent self-circularization events and were thus expected to have circular chromosomes with different fusion points. Southern blot analyses revealed that the telomeric repeat was completely absent in all eight derivative clones (data not shown).

\section{Subtelomeric regions of circular chromosomes behave as endogenous telomeres in the horsetail stage}

It is known that telomeres play an important role during meiosis, particularly in fission yeast (Hiraoka 1998; Ishikawa and Naito 1999). Fission yeast telomeres are clustered near the SPB (spindle pole body, equivalent to the centrosome in higher eukaryotes) transiently during the period called the "horsetail stage" that precedes meiosis I (Chikashige et al. 1994; Scherthan et al. 1994). It was previously reported that the telomere-SPB interaction and spore viability are markedly reduced in taz1 and rap1, mutants of the telomeric DNA-binding protein and the Tazl-interacting protein, respectively (Cooper et al. 1998; Nimmo et al. 1998; Chikashige and Hiraoka 2001; Kanoh and Ishikawa 2001). Although the trt1 derivative clones that lacked telomeric repeats mated and performed cell fusion and karyogamy normally, all of them showed markedly reduced spore viability (Nakamura et al. 1998; data not shown). Those studies suggested that the telomeric DNA-Taz1-Rap1 interaction is important for telomere association with SPB and for productive meiosis.

We first examined whether the horsetail-stage-specific nuclear organization /the juxtaposition of telomeric/subtelomeric regions to the SPB) was affected in the derivative clones. For this purpose, we used $\cos 212$, which hybridizes with the fission yeast subtelomeric regions at both ends of Chromosomes I and II, but not with those of Chromosome III (Funabiki et al. 1993). We found that fragments hybridizing with $\cos 212$ were retained at the fusion points of circular Chromosomes I and II (see below), and used this probe in FISH (fluorescence in situ hybridization) experiments to detect the fusion points of these chromosomes. The SPB was simultaneously detected by the indirect immunofluorescence (IF) technique using the anti-Sad1 antibody (Hagan and Yanagida 1995). In wild-type cells, one cos212 signal was observed near the SPB signal in most horsetail-stage cells, which reflected the telomere clustering at $\mathrm{SPB}$, as reported previously (Fig. 1A, wild type; Cooper et al. 1998; Nimmo et al. 1998). Surprisingly, the cos 212 signals were frequently associated with the SPB signal although the fusion points did not contain any telomeric repeats (Fig. 1A, clone a). The frequency of $\cos 212-$ SPB association differed among independent derivative clones and was consistent in a particular clone in repeated experiments. We grouped the eight derivative clones according to the frequency of cos212-SPB association (Fig. 1B). Type A, which includes clones a, c, d, e, and h, consistently showed high frequencies of $\cos 212-\mathrm{SPB}$ association: The percentage of cells having a single cos212 signal close to an SPB signal was $>50 \%$ in Type A clones. Type B, the only member of which is clone $b$ in this series of experiments, showed a low frequency of cos212-SPB association. No cos212 signal was observed near an SPB signal in more than $80 \%$ of Type B cells. Type AB, which includes clones $f$ and $g$, showed phenotypes intermediate of those of Types A and B. In this type of cell, one cos 212 signal was frequently $(>50 \%)$ located close to an SPB signal, whereas simultaneously one or two cos 212 signals were distant from it. Therefore, five out of eight randomly chosen derivative (circular-chromosome-possessing) clones showed significantly high frequencies of cos212-SPB association (Type A clones). Importantly, these phenotypes were very stable during the extensive propagation of each derivative clone.

The subtelomeric region of fission yeast is composed of different classes of repeats present in low-copy numbers, which are collectively called TAS (telomere-associated sequence; Sugawara 1989). Four telomeres of Chromosomes I and II and one telomere of Chromosome III contained TAS in the yeast strain used in this study 

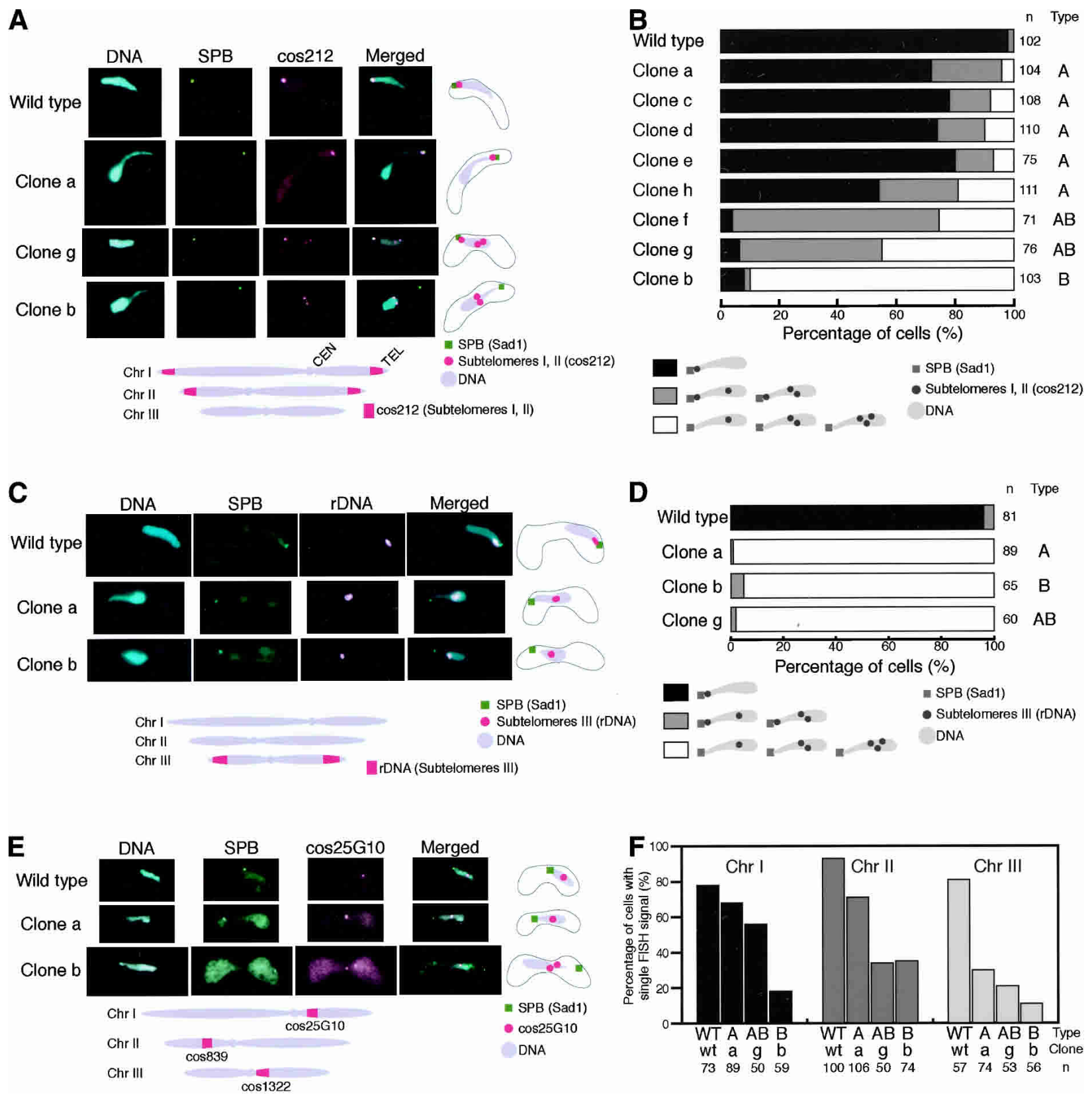

Figure 1. Subtelomeric regions of circular chromosomes behave like endogenous telomeres in the horsetail stage. (A) Cos212-SPB association in the horsetail stage of derivative cells. Cells in the horsetail stage were examined simultaneously by FISH analysis using the $\cos 212$ probe, which detects subtelomeric DNA of Chromosomes I and II (cos212), and by IF analysis using anti-Sad1 antibodies that detect SPB. Cos212-reactive sequences are present at the four ends of Chromosomes I and II, but absent on Chromosome III (lower panel). (B) Summary of cos212-SPB association frequencies in eight independent derivative clones (a-h) as well as in wild-type cells. Cos212-SPB associations were classified in terms of FISH results according to the criteria shown below. $(C, D)$ The fusion points of circular Chromosome III do not cluster at the SPB in the horsetail stage in Types A, B, and AB clones (clones a, b, and g, respectively). The subtelomeric regions of Chromosome III (rDNA) were detected by FISH analysis using the rDNA probe (lower panel). rDNA-SPB associations were classified in terms of FISH results according to the criteria shown below. $(E, F)$ Efficiencies of homolog pairing of circular chromosomes in Types A, B, and AB clones (clones a, b, and g, respectively). The cosmid clone that detects Chromosome I (cos25G10), II (cos839), or III (cos1322) was individually used as a FISH probe. The percentages of cells in which FISH signals appeared as a single dot or two closely located dots are shown.

(M. Sadaie, unpubl.), and these TAS-positive subtelomeres showed similar sequence organization (Sugawara 1989; M. Sadaie, J. Kanoh, and F. Ishikawa, unpubl.). We found that circular Chromosome III in the $\Delta$ trt1 background lacked TAS. As rDNA clusters are mapped near both ends of Chromosome III, we analyzed the SPB as- 
sociation of the fusion points of circular Chromosome III using rDNA as a FISH probe. In contrast to the wild type in which the rDNA signal was always detected near the $\mathrm{SPB}$, all derivative clones examined, irrespective of Type $\mathrm{A}, \mathrm{B}$, or $\mathrm{AB}$, did not show any significant rDNA-SPB association (Fig. 1C,D). Collectively, we conclude that the fusion points of circular Chromosomes I and II but not those of Chromosome III remain associated with SPB in some derivative clones.

Efficiency of homologous chromosome pairing correlates with that of subtelomere-SPB association

The telomere-SPB association promotes the pairing of homologous chromosomes in meiosis (Hiraoka 1998; Niwa et al. 2000). To determine if the remaining association between the SPB and the fusion points of circular Chromosomes I and II has any biological consequences, we indirectly measured the efficiency of homologous chromosome pairing in the derivative clones using FISH (Fig. 1E,F). Three cosmid clones, each mapped on the arm region of each of the three chromosomes (Fig. 1E), were used as FISH probes. When a single FISH signal was observed in horsetail-stage cells, it was inferred that the cognate homologous chromosomes were paired. When two signals were found, we surmised that chromosome pairing did not occur. Figure $1 \mathrm{E}$ shows representative FISH data, and the results are summarized in Figure 1F. Circular Chromosome III pairing was inefficient in Types A, B, and AB clones. Type A clones showed efficient pairing of circular Chromosomes I and II. In contrast, Type AB clones showed efficient pairing of circular Chromosome I but not of II. Type B clones were defective in pairing of all three chromosomes. Altogether, these results led us to hypothesize that Types $A, A B$, and $\mathrm{B}$ clones retained the subtelomere-SPB (fusion point) association ability in Cromosomes I and II, Chromosome I only, and none of the three chromosomes, respectively. This hypothesis suggested that the $\cos 212$ signals distant from the SPB signal in Type AB clones (Fig. 1A,B) should represent the subtelomeres of circular Chromosome II. The strong correlation between the ability to undergo subtelomere-SPB association and chromosome pairing suggests that the fusion points that colocalize with SPB are functional in terms of promoting homologous chromosome pairing.

\section{Larger regions of TAS are lost in Type B than in Type A clones}

To understand how Types A, B, and AB clones showed differential levels of residual telomere function, we analyzed the DNA structure at the fusion points of these derivative clones. pNSU70, pNSU56, and pB15E1, which contain TAS regions, are three overlapping clones (Sugawara 1989; Wood et al. 2002), and a composite physical map is shown in Figure 2A. Although this map does not show the exact structure of the TAS regions in the strain used in this study, it provides a rough idea of the orga- nization and the order of appearance of the sequences used in this study. The $12.5-\mathrm{kb}$ centromeric end of pB15E1 also overlaps with the telomeric end of $\cos 212$, although a small insertion, which is most likely due to polymorphism, was found in $\cos 212$. Thus, a $40-\mathrm{kb}$ contiguous subtelomeric sequence was deduced from pNSU70, pNSU56, pB15E1, and cos212. We first examined whether any occult telomeric repeat was present in this region. Although the fission yeast telomeric repeat is variable, the GGTTAC repeat occurs most frequently (Cooper et al. 1997). Tazl was reported to bind poorly to a single copy of GGTTAC and efficiently to two tandem repeats of GGTTAC (Spink et al. 2000). We found no two consecutive repeats of GGTTAC in either strand of these regions except for the native telomeric repeats. Moreover, when the frequency of GGTTAC appearance was determined quantitatively on a kilobase-DNA basis, we did not find any local accumulation of the telomeric repeat, compared with other arbitrary internal sequences (cos25G10, cos839, cos1322, and $n m t 1^{+}$promoter$n m t 1^{+}-n m t 1^{+}$terminator; a maximum of 2 repeats $/ \mathrm{kb}$ in all cases). Variant telomeric repeats, such as TTACAG, TTACGG, and TTACACG (Sugawara 1989), which were subjected to the same analysis, yielded essentially the same results. Accordingly, we conclude that the $40-\mathrm{kb}$ subtelomeric region in fission yeast does not significantly contain telomeric repeats to which Tazl binds.

A sequence called TAS2 is located at a region 2-6 kb centromere-proximal to the telomeric repeats (Fig. 2A). We found that TAS2 is not present in all of the eight derivative clones (data not shown) as reported previously (Nakamura et al. 1998), suggesting that the terminal regions of at least $\sim 5 \mathrm{~kb}$ were lost in all of the derivative clones during the formation of circular chromosomes (data not shown). We next examined ClaI-digested genomic DNA using fragment 562 as a probe (Fig. 2A,B). Sequences hybridizing with fragment 562 are present at all ends of Chromosomes I and II, and at least one end of Chromosome III (Sugawara 1989; M. Sadaie and F. Ishikawa, unpubl.). Therefore, the relative intensities of this band in the different derivative clones reflect the extent of subtelomere deletion during circular chromosome formation. To analyze the result quantitatively, we simultaneously hybridized the blot with an his $1^{+}$probe as an internal control. The signal intensities of the 562 band and the $h i s 1^{+}$band were calculated, and the relative intensity of the 562 band to the his $1^{+}$band was determined by setting the value obtained with the wild type as 1 (Fig. 2B). The relative intensities of the 562 band in Type A clones were approximately half of that in the wild type. Type B (clone b) did not show any 562 band. Type AB clones showed lower relative intensities of the 562 band (one-fourth of that in the wild type). Impressively, the relative intensity values of the 562 band correlated well with the relative efficiencies of the subtelomere-SPB association in all of the eight examined derivative clones. The correlation confirmed the quantitative nature of the analyses and supported the idea that the greater the loss of the subtelomeres, the fewer telomere-associated functions remained. Type B clone was further analyzed by 
A
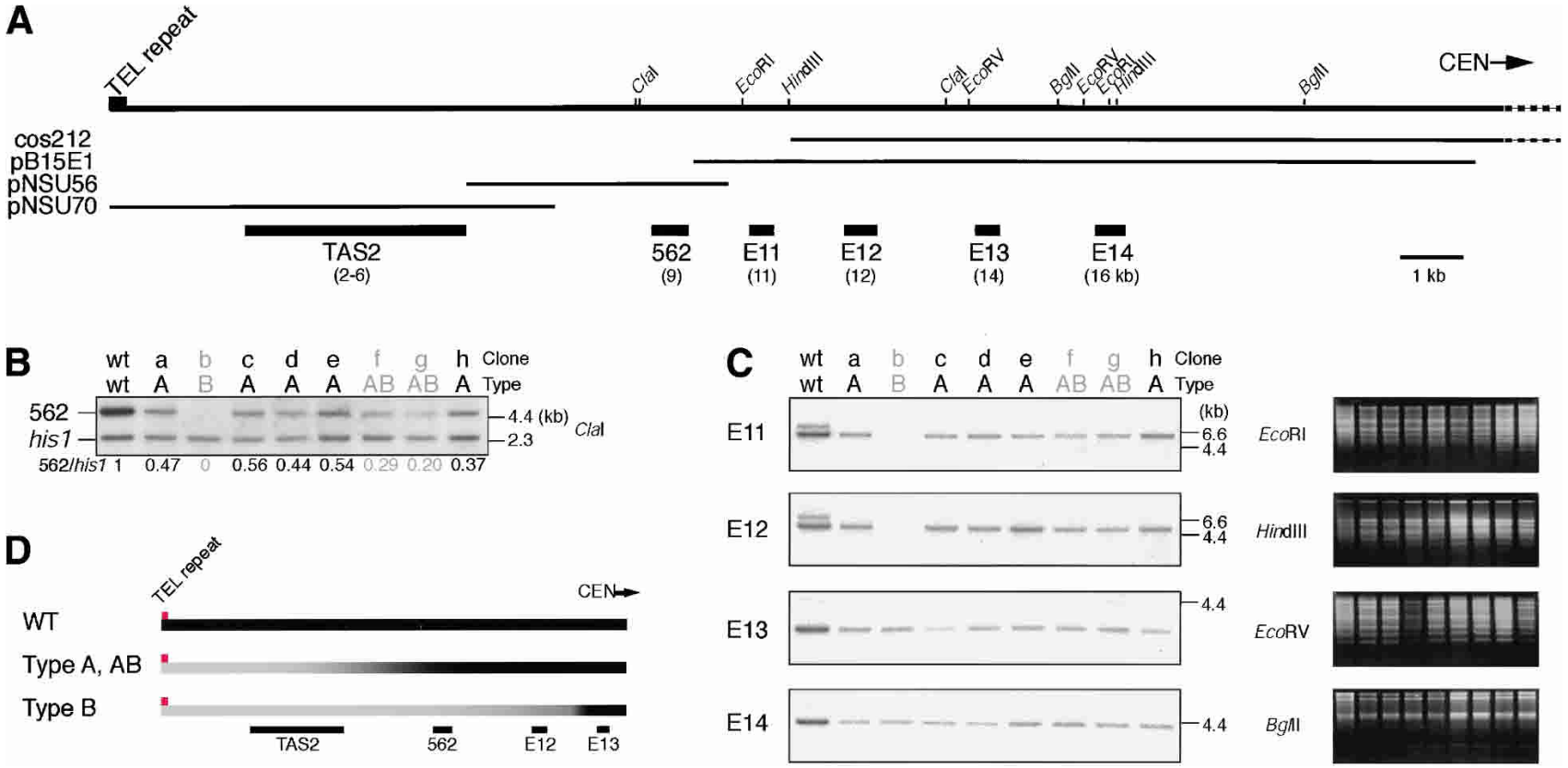

Figure 2. Larger regions of TAS are lost in Type B clones than in Type A clones. (A) A composite physical map of pNSU70, pNSU56, pB15E1, and cos212, which are overlapping clones containing fission yeast telomere-associated sequences (TASs). The locations of the probes used in Southern hybridization are indicated by thick bars, together with the distance (in kilobases) of the probe from the telomeric repeats (parentheses). Restriction sites relevant to the experiment are shown. $(B)$ Relative copy numbers of 562-hybridizing sequences in derivative clones. ClaI-digested genomic DNA was simultaneously hybridized with the 562 probe and the his 1 probe. The calculated relative intensities of the 562 band to the his1 band are shown at the bottom. $(C)$ Genomic DNAs digested with indicated restriction enzymes were hybridized with the E11, E12, E13, or E14 probe. Ethidium-bromide-stained DNAs are shown as a loading control (right). In wild-type cells, doublets were detected with probes E11 and E12. The upper band is lost in all derivative clones, and may be derived from the Chromosome III subtelomere. $(D)$ Schematic representation of subtelomeric deletion in Types A, AB, and B clones.

Southern hybridization using more internal probes, E11E14, which are located $11 \mathrm{~kb}, 12 \mathrm{~kb}, 14 \mathrm{~kb}$, and $16 \mathrm{~kb}$ from the telomeric repeats, respectively (Fig. 2A). Whereas the E11-E14 probes hybridized with Type A and Type AB DNAs, the E11 and E12 probes failed to hybridize with Type B DNA, but the E13 and E14 probes did (Fig. $2 \mathrm{C}$ ), indicating that the Type B clone lacks the $\sim 12$ $\mathrm{kb}$ subtelomeric region. These results suggest that the deletion of the subtelomeric region in all types of cells is contiguous from the terminal region. The degree of terminal truncation in each type of clone is schematically shown in Figure 2D.

\section{Taz1 associates with TAS in addition} to telomeric repeats

It is generally believed that telomeric repeats play a primary role in telomere establishment (telomere seeding) and inheritance (Gottschling et al. 1990; Sandell and Zakian 1993). However, the results described above indicate that the telomere function in meiosis is maintained in the absence of telomeric repeats. In fission yeast, Taz1 binds to telomeric repeats (Cooper et al. 1997) and recruits Rap1 to execute telomere function (Chikashige and Hiraoka 2001; Kanoh and Ishikawa 2001). We therefore examined whether Tazl associates with TAS in the derivative clones (Fig. 3). Because it is not known with what extent of DNA Tazl associates, we first examined Tazl-interacting sequences by the ChIP (chromatin immunoprecipitation) method in wild-type cells.

The HA-tagged $\operatorname{taz} 1^{+}$construct was integrated into the wild-type locus under the control of its native promoter. HA tagging had a negligible effect on Tazl function (data not shown). In this experiment, we paid particular attention to controlling the size of the sonicated DNA so that its length distribution would be $<1 \mathrm{~kb}$ (Supplementary Fig. S1A). Because Taz1 binds to $\sim 300$-bp telomeric repeats with high affinity, it was expected that the telomere-flanking region called 70TEL (Sugawara 1989), which is located within $1 \mathrm{~kb}$ from the proximal border of telomeric repeats (Fig. 3A), would be coimmunoprecipitated by anti-HA antibodies as part of continuous DNA with telomeric repeats. As expected, a PCR product derived from 70TEL was efficiently and specifically detected in the Taz1-HA complex from wild-type cells (Fig. $3 \mathrm{~B})$. The $K$ region located between mat2 and mat 3 loci forms heterochromatin, but does not contain telomeric repeats (Grewal and Klar 1997). This region was not precipitated by anti-HA antibodies in Taz1-HA cells, indicating that Tazl is not a general heterochromatin protein, as shown previously (Kanoh and Ishikawa 2001). Next, we examined several regions that are positioned 
Sadaie et al.

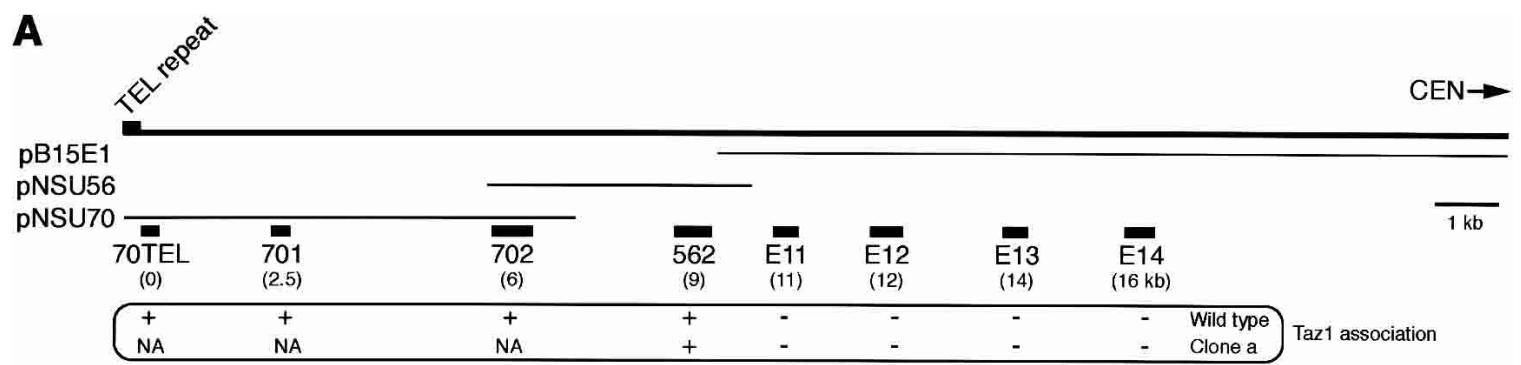

B
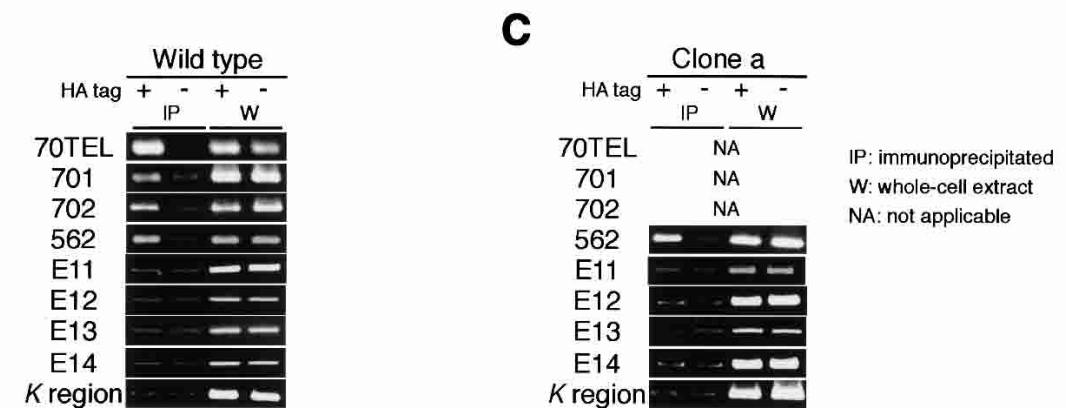

Figure 3. Tazl interacts with TAS in both wild-type and Type A derivative clones. (A) Approximate and relative positions of the PCR products used to detect Tazl-TAS interaction in $B$ and $C$. The results shown in $B$ and $C$ are summarized below the map. $(B)$ Results of the ChIP assay for Tazl-HA and TAS interactions. As control, wild-type cells in which Tazl was not tagged (HA tag -) were used in addition to wild-type cells expressing HA-tagged Tazl (HA tag +). Whole-cell extracts (W) were analyzed to monitor extraction efficiencies. The $K$ region, located between mat2 and mat3 loci, was used as a negative control. (C) Fragments 562 and E11-E14 were analyzed as in $B$ using Type A cells (clone a) expressing HA-tagged or HA-untagged Taz1.

more centromere-proximally in TAS. We found that anti-HA antibodies precipitated three regions in TAS, namely, 701, 702, and 562, which are located $2.5 \mathrm{~kb}, 6$ $\mathrm{kb}$, and $9 \mathrm{~kb}$ from the telomeric repeats, respectively (Fig. 3A,B). Because these sequences are located $>1 \mathrm{~kb}$ away from the telomeric repeats, it was unlikely that they were precipitated as part of continuous DNA with telomeric repeats. In contrast, anti-HA antibodies did not precipitate the more centromere-proximal regions, E11, E12, E13, and E14 (Fig. 3A,B). Similar results were obtained in experiments that detected these DNAs in precipitates by direct hybridization without PCR amplification (Supplementary Fig. S1B). These results suggest that in wild-type cells, Tazl accumulates at the TAS regions spanning over at least $9 \mathrm{~kb}$ from the telomeric repeats. The Taz1 accumulation at the subtelomere appears to occur contiguously from telomeres, and is terminated at a relatively narrow region between fragments 562 and E11. By inspection, however, we did not notice any characteristic nucleotide sequence between the 562 and E11 regions. We next examined the Tazl-interacting sequences in a Type A clone (clone a) in which the endogenous $\operatorname{taz} 1^{+}$was replaced with the HA-tagged $t a z 1^{+}$. Fragments 70TEL, 701, and 702 were absent in the genome of clone a (data not shown). We found that Taz1 accumulated at 562 and not at more internal regions (E11, E12, E13, and E14) in clone a (Fig. 3C). The detection of Taz1-HA at fragment 562 in clone a was unexpected, because it indicated that significant amounts of Tazl remained bound to subtelomeric regions even after its binding sites with the highest affinity (telomeric repeats) were lost. These results indicate that the Taz1-
TAS association is retained in the absence of telomeric repeats.

\section{Epigenetic inheritance of SPB-subtelomere association} in Type A clones

ChIP experiments suggested the important role played by Tazl in maintaining telomere function in Type A clones. To examine this possibility genetically, taz $1^{+}$ was disrupted in wild-type, Type A (clone a), and Type B (clone b) clones. In the wild-type background, $\operatorname{taz} 1^{+}$disruption caused a marked reduction in the frequency of subtelomere-SPB association, as previously reported (Fig. 4A, top; Cooper et al. 1998; Nimmo et al. 1998). We found that $t a z 1^{+}$disruption also markedly reduced the frequency of cos212-SPB association in Type A clone [Fig. 4A (middle), B]. In contrast, $t a z 1^{+}$disruption did not significantly change the low frequencies of $\cos 212-\mathrm{SPB}$ association in Type B clones (Fig. 4A, bottom). Then, we reintroduced $t a z 1^{+}$into the $t a z 1^{+}$-deleted $(\Delta t a z 1)$ Type A clone to determine whether the $\cos 212-S P B$ association was restored or not. We integrated $\operatorname{taz} 1^{+}-H A$ at the native $t a z 1^{+}$locus of the $\Delta t a z 1$ clone. The expression of Tazl-HA was confirmed by immunoblotting with antiHA antibodies (data not shown). The production of Taz1HA in $\Delta$ taz1 (generated from wild-type cells and maintaining linear chromosomes) rescued the defective $\cos 212-\mathrm{SPB}$ association to the level found in wild-type cells (Fig. 4A, top), indicating that Tazl-HA is functional. In contrast, we found that the defective cos212SPB association in $\Delta$ taz1 Type A clones was not rescued by the Taz1-HA reintroduction (two independent clones 
A

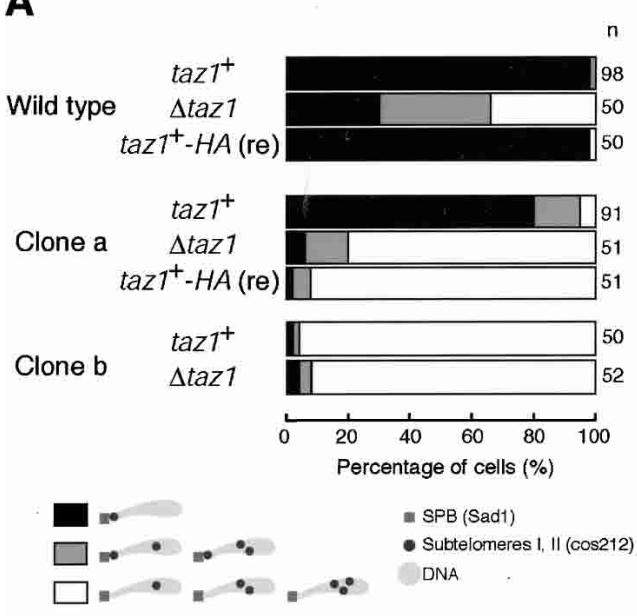

B
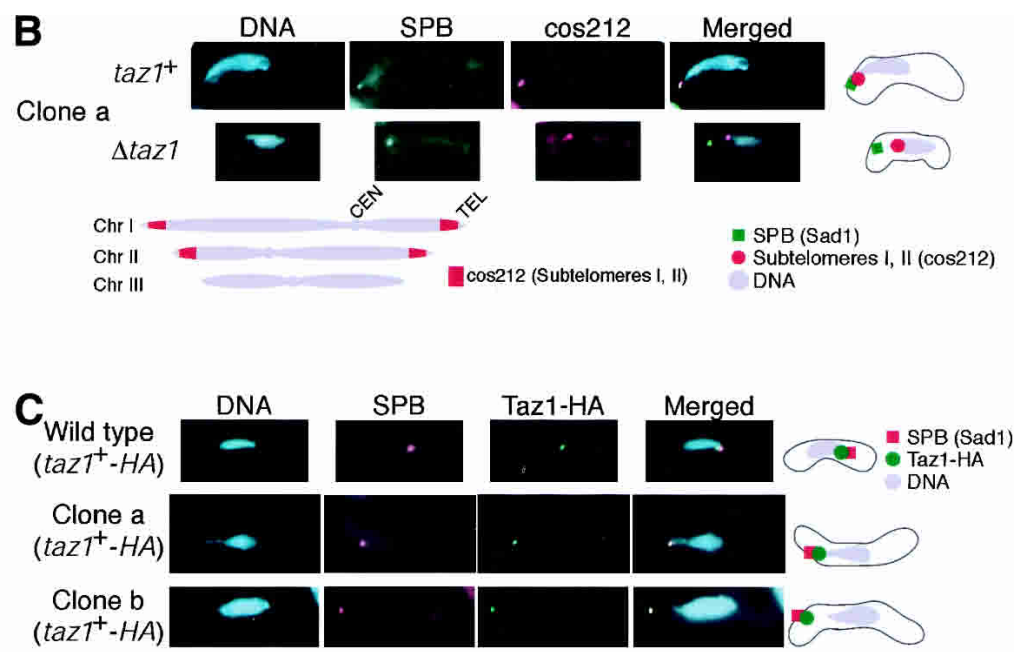

D

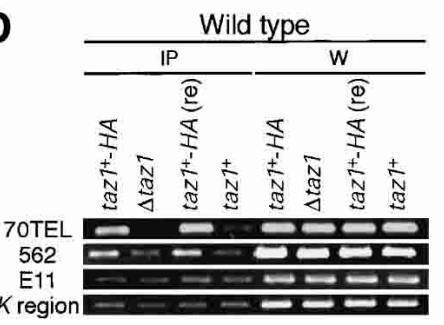

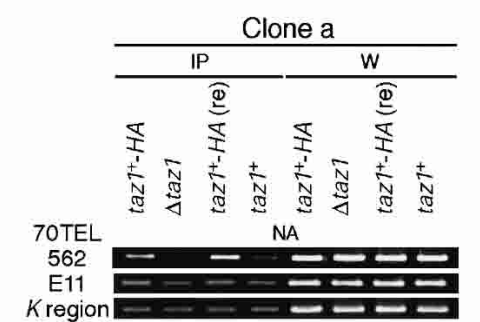

\begin{abstract}
IP: immunoprecipitated
W: whole-cell extract

NA: not applicable
\end{abstract}

Figure 4. Taz1-dependent epigenetic inheritance of subtelomere-SPB association in Type A clones. $(A)$ Cos212-SPB association is disrupted in the $\Delta t a z 1$ Type A clone and is not restored by the reintroduction of $\operatorname{taz} 1^{+}$. Cos212-SPB association frequencies of indicated strains in the $\Delta t a z 1$ background are shown as in Figure 1B. $t a z 1^{+}-H A$ (re) indicates Taz1-HA-expressing strain in which $t a z 1^{+}-H A$ was integrated at the $t a z 1^{+}$locus of each $\Delta t a z 1$ strain. (B) Typical images of Type A cells (clone a) with or without taz1 $1^{+}$in the horsetail stage. $(C)$ Tazl localizes at the leading tip of the horsetail nuclei in wild-type and Type A cells, whereas it dissociates from DNA in Type B cells. Taz1-HA and SPB were simultaneously stained with anti-HA antibodies and anti-Sad1 antibodies, respectively. (Top) Wild type. (Middle) Type A clone. (Bottom) Type B clone. (D) The Tazl-subtelomere association is restored by the reintroduction of $t a z 1^{+}$into $\Delta t a z 1$ Type A clones. ChIP experiments were conducted as described in Figure 3. $\operatorname{taz} 1^{+}-H A$ indicates strains in which the endogenous $t a z 1^{+}$was tagged by HA, and $t a z 1^{+}-H A(\mathrm{re})$ indicates $\Delta t a z 1$ strains in which $\operatorname{taz} 1^{+}-H A$ was integrated to the original taz1 $1^{+}$ locus.

were tested; Fig. 4A, middle). We also confirmed that the fusion points in clone a were not structurally altered by the procedures of deletion and reintroduction of $t a z 1^{+}$ (data not shown). These results invalidated the hypothesis that the proficient subtelomere-SPB association in Type A clones is genetically determined, and that the remaining TAS in Type A clones has an intrinsic ability to associate with SPB. Rather, it is highly likely that Type A clones maintain the subtelomere-SPB association in an epigenetic manner.

We further examined the localization of Taz1-HA in the derivative clones. Taz1-HA was observed in wildtype cells as a single and discrete spot at the leading tip of the horsetail nuclei, where SPB was also present (Fig. 4C, top row). In Type A cells (clone a) harboring the HA-tagged taz1 $^{+}$at the native locus [not $\operatorname{taz} 1^{+}-H A$ (re)], we found that Taz1-HA was frequently $(78 \%)$ localized at the leading tip of the horsetail nuclei, with which the SPB was closely associated. Because the leading tip of the horsetail nuclei in Type A cells colocalizes with $\cos 212$ (Fig. 1), this result supports the ChIP data, and together indicates that Tazl associates with TAS in Type A clones. In contrast, in $75 \%$ of Type B cells, Taz1-HA was localized at the SPB as a single spot, and this signal was completely dissociated from the nucleus (Fig. 4C, bottom row). This result was interpreted as Tazl associating with the SPB without DNA binding, probably through a protein-protein interaction with Rap1 (Kanoh and Ishikawa 2001). Altogether, these results indicate that the ability of subtelomeres to cluster at the SPB in the absence of telomeric repeats is inherited epigenetically in a manner dependent on Tazl bound to TAS.

\section{Subtelomeres have an independent element recruiting Taz1}

ChIP and IF results suggested that Tazl remaining at TAS plays an essential role in the subtelomere-SPB association in Type A cells (Figs. 3, 4A,B,C). It is possible that the subtelomere-SPB association remains inacti-

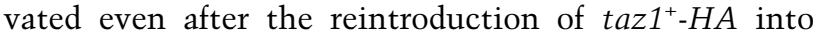
$\Delta t a z 1$ Type A cells because the re-expressed Tazl was mislocalized from TAS. Surprisingly, however, we found that Tazl bound to the 562 region when $\operatorname{taz} 1^{+}-H A$ was 
Sadaie et al.

reintroduced into $\Delta$ taz1 Type A cells (Fig. 4D). This suggests that the TAS region remaining in Type A but not Type B clones has an independent element recruiting Taz1, and that the Taz1-TAS association in Type A clones is genetically inherited. Because the subtelomereSPB association was not restored when Tazl was reassociated with TAS by the reintroduction of $\operatorname{taz} 1^{+}-H A$, Tazl at TAS on its own is not sufficient, even though essential, for mediating the subtelomere-SPB association. There should be another factor(s) that is epigenetically associated with subtelomeres and forms together with Tazl a functional complex required for the subtelomereSPB association.

Swi6 is present at fusion points of circular chromosomes

The Tazl reintroduction did not restore the subtelomere-SPB association in clone a $\Delta t a z 1$, although Taz1 reassociated with the 562 region upon the reintroduction. This suggests that there is another factor(s) missing in clone a $\operatorname{taz} 1^{+}-H A(\mathrm{re})$ and responsible for the subtelomere-SPB association. This factor may be epigenetically maintained in the original clone a, but lost from subtelomeres once $t a z 1^{+}$is deleted. Swi6 is a fission yeast protein homologous to the heterochromatin protein HP1 in higher eukaryotes. It is known that Swi6 is present in three classes of heterochromatin in fission yeast, namely, heterochromatin in the mating-type locus, cen- tromeres, and telomeres (Ekwall et al. 1995; Nakayama et al. 2000), and is important for gene silencing at these loci (Allshire et al. 1995). Therefore, we surmised that Swi6 is a candidate for the missing factor in clone a taz1+-HA (re). We examined the localization of Swi6 in the derivative clones. For this purpose, we constructed a clone in which the swi $6^{+}$-GFP fusion construct was integrated into the $s w i 6^{+}$locus. It was reported that the C-terminal-GFP-tagged Swi6 protein distributes normally at heterochromatin, although it may not be fully functional (Wang et al. 2000). Therefore, we could analyze the subcellular localization of Swi6 in cells expressing Swi6-GFP. In wild-type vegetative cells, we observed several GFP dot signals, as previously reported (Ekwall et al. 1995). Some of those signals were colocalized with FISH signals obtained using a centromere-specific probe, as expected (data not shown). We next examined the relative positions of Swi6-GFP and $\cos 212$. In wild-type vegetative cells, most cos 212 signals were found to overlap with Swi6-GFP signals (98\%, $n=50$; Fig. 5A, top). Cos212 signals were colocalized with Swi6-GFP signals in Type A vegetative cells at the same frequency as that in wild-type cells $(98 \%, n=51$; Fig. 5A, middle). These results suggest that Type A cells maintain at least part of the telomere-specific heterochromatin structures (Taz1 and Swi6 association) at TAS in vegetative (Figs. 3, 5A) and meiotic cells (Figs. 1, 4). The high frequency of Swi6$\cos 212$ colocalization in Type A cells indicates that the Swi6 localization at the fusion point is maintained inde-

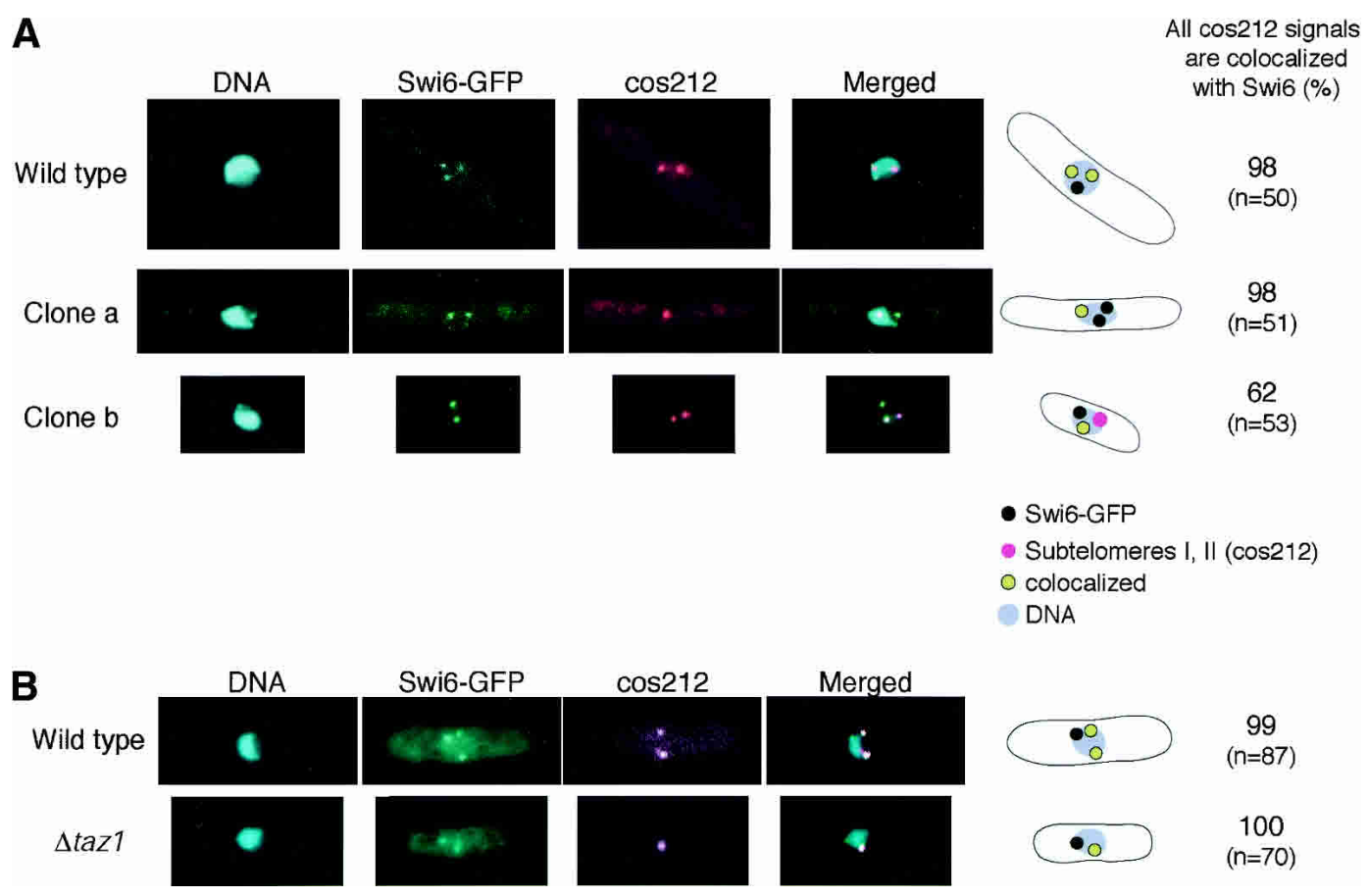

Figure 5. (A) Swi6 is present at the fusion points of circular chromosomes. The subtelomeric DNAs of Chromosomes I and II (cos212) were detected with $\cos 212 \mathrm{FISH}$, and the frequencies of all $\cos 212$ signals overlapping with Swi6-GFP signals were calculated for wild-type, Type A (clone a), and Type B (clone b) cells. (B) Tazl is dispensable for Swi6 localization at telomeres. Frequencies of Swi6-GFP-cos 212 colocalization in wild-type cells (having linear chromosomes) and $\Delta$ taz1 (originated from the wild-type) cells are shown as in $A$. 
pendently of telomeric repeats. Interestingly, Type B clones showed a higher frequency of Swi6-cos212 colocalization (62\%, $n=53$; Fig. 5A, bottom) than that expected from the low frequency of Taz1-DNA association. This suggests that the association of Swi6 with subtelomeres may be largely independent of Taz1 binding to DNA. To examine this possibility more directly, we examined Swi6 localization in $\Delta$ taz1 cells and found that Swi6 associated with $\cos 212$ in $\Delta$ taz1 (having normal linear chromosomes) at a frequency as high as in wildtype cells (Fig. 5B). Therefore, the finding that Swi6 binds to telomeres and subtelomeres in a manner independent of Tazl indicates that Swi6 is unlikely to be the missing protein in clone a $\operatorname{taz} 1^{+}-H A(\mathrm{re})$.

\section{Discussion}

In this study, we found that at least part of the telomereassociated chromatin structure (Tazl binding) and function (telomere/subtelomere-SPB association at the horsetail stage, leading to homologous chromosome pairing) is stably inherited in the absence of telomeric repeats. However, the mechanisms responsible for the inheritance are different. Tazl recruitment to subtelomeres is maintained by a genetic mechanism, whereas subtelomere-SPB association in Type A clones is maintained by an epigenetic mechanism, suggesting that (1) the subtelomeres function as a genetic element that backs up telomeric repeats to ensure the telomere structure when the telomeric repeats become very short or are lost; and (2) the epigenetic mechanism contributes to the maintenance of the telomere-associated function, probably by inheriting a higher-ordered structure required for the function in the absence of telomeric repeats. Importantly, we showed that the functional complex responsible for the telomere-SPB association is not re-established in the absence of telomeric repeats in the $\operatorname{taz} 1^{+}$ deletion/reintroduction experiment, underscoring the essential role played by the telomeric repeats in establishing the functional complex. Our observation closely parallels the situation at the fission yeast mating-type locus, and both indicate that the establishment of heterochromatin by a cis-element and the subsequent maintenance through an epigenetic mechanism are distinguishable (Hall et al. 2002). We surmise that these mechanisms also operate in diverse groups of heterochromatin in other species.

\section{Genetic inheritance of Taz1 at subtelomeres in the absence of telomeric repeats}

Perhaps one of the most striking and intriguing findings in this study is that Taz1, a well-characterized DNAbinding protein highly specific to telomeric repeats (Cooper et al. 1997; Vassetzky et al. 1999; Spink et al. 2000), accumulated at the fusion points of Type A circular chromosomes where there are no telomeric repeat. In budding yeast, it has been shown by ChIP analysis that the telomeric DNA-binding protein, Rap1, and its bind- ing partners associate with subtelomeric regions in addition to telomeric repeats (Strahl-Bolsinger et al. 1997; Lieb et al. 2001; Smith et al. 2003). This result was interpreted as follows: Rap1, which directly associates with telomeric repeats, indirectly interacts with the subtelomeres via the looping back of chromosome ends (Strahl-Bolsinger et al. 1997). This interpretation is not applicable to our case, however, because circular chromosomes do not have any telomeric repeats.

Several independent lines of evidence support the notion that Tazl is recruited to subtelomeres by a genetic element present in TAS in the absence of telomeric repeats. First, we demonstrated that Taz1 in Type A cells bound to subtelomeric DNA probed by the 562 primer set in the ChIP experiment (Fig. 3). A consistent result was also obtained in our IF study demonstrating that Taz1, SPB, and subtelomeres were all colocalized in Type A cells (Figs. 1, 4C). In contrast, Tazl in Type B cells was localized not at subtelomeres but at the SPB in the same IF experiment. Therefore, it is highly likely that Tazl does not bind to the remaining subtelomeric region in Type B cells. Importantly, Type B cells lost larger subtelomeric regions than Type A cells (Fig. 2), suggesting that the subtelomeric region missing in Type B cells but present in Type A cells contains a genetic element that recruits Taz1. Second, when functional taz1+-HA was reintroduced into taz1-deleted Type A clones, we observed the reappearance of Tazl associating with region 562 in the ChIP assay (Fig. 4D). This result strongly suggests that there is a genetic element that recruits Tazl in the remaining TAS in Type A clones. Although we do not know the identity of the sequence at present, we predict that the DNA sequence in the proximity of region 562 is responsible for this element, because the 562 primer set was the only probe that detected Tazl binding in our ChIP assay (Fig. 3).

We did not find two consecutive telomeric repeats or related sequences to which Tazl reportedly most strongly binds in the 40-kb composite TAS sequences retrieved from the database and the sequence of pNSU56 (T.M. Nakamura, pers. comm.). It is possible that Taz1 binds to a previously unrecognized sequence motif that is totally unrelated to the telomeric repeats. Such a second Tazl-binding motif may be present in TAS. Finally, Tazl may be recruited to TAS in Tazl-reintroduced Type A clones, not by the direct Tazl binding to DNA but by a protein-protein interaction(s). Tazl is known to physically interact with Rap1 and Rif1, and probably with some other proteins (Chikashige and Hiraoka 2001; Kanoh and Ishikawa 2001). Therefore, a genetic element in TAS may first bind to these Tazl interactors and then indirectly recruit Taz1. Further studies are necessary to elucidate the molecular mechanism governing the telomeric-repeat-independent Taz1 recruitment to TAS.

Some strains lack TAS at both or one of the two subtelomeric regions of Chromosome III; nevertheless, both ends of Chromosome III maintain functional telomeres in all aspects including the telomere-SPB association in the horsetail stage. We therefore think that telomeric repeats are the primary domain responsible for Tazl 
binding, and that TAS is an auxiliary but independent domain that recruits Taz1. Nevertheless, TAS may play the role of a backup system through a variety of mechanisms (Lundblad and Blackburn 1993; Nakamura et al. 1998) when telomeric repeats are insufficient, thereby ensuring the continuity of species.

Epigenetic inheritance of subtelomere-SPB association in the absence of telomeric repeats

All functional chromosome domains including telomeres are huge molecular complexes consisting of a large number of proteins and cognate DNA. It is also probable, albeit not proven, that these molecular complexes are not a simple assembly of components but a highly organized structure. Typically, these component proteins form the complex via multivalent protein-protein interactions. Those ordered protein interactions may not take place spontaneously by supplying all components (Ekwall et al. 1997; Nakayama et al. 2000). However, once formed, the organized complex may show a robust nature of maintenance as exemplified by the metastable inheritance of an epigenetic state. When the complex is destroyed by, for example, inactivating an essential component, it may not be formed easily even after the missing component is resupplied. Exactly what we expect from these arguments is observed in this study.

We found that the subtelomere-SPB association is proficient in Type A clones that lack telomeric repeats. The subtelomere-SPB association in the horsetail stage is presumably accomplished by a complex involving many proteins and telomeric DNA. Tazl is an essential component of this complex, because the deletion of $t a z 1^{+}$led to the inactivation of the association process, in both wild-type and derivative cells. However, the reintroduction of the functional $\operatorname{taz} 1^{+}-H A$ did not rescue the low efficiencies of subtelomere-SPB association in the Type A $\Delta$ taz1 clone, whereas it did in $\Delta$ taz 1 derived from wildtype cells. This indicates that the complex responsible for the subtelomere-SPB association in Type A clones is maintained epigenetically. Moreover, it is suggested that the epigenetically maintained complex is robust in terms of stable inheritance during numerous generations, but once inactivated by removing Taz1, it is not spontaneously re-established even after Tazl is resupplied. Because Tazl (and presumably its interacting factors) is colocalized at SPB in Type B cells (Fig. 4C), it is likely that the complex responsible for SPB-Tazl association is maintained in the absence of DNA binding of Taz1. This complex is expected to bind to subtelomeres and achieve the SPB-subtelomere association in Type A but not in Type B clones.

Although the reintroduction of $\operatorname{taz}^{+}$restored Tazl binding to TAS, it alone was not sufficient to restore the functional complex. This observation re-emphasizes the importance of telomeric repeats in the establishment of the functional telomere complex and suggests that some unique features conferred by telomeric repeats, other than simply providing a scaffold to Taz1, are required for telomere establishment. It is possible that other telomere factors uniquely binding to either single-stranded or double-stranded telomeric repeats are necessary for the process. However, when we ectopically integrated an array of $\sim 300-b p$ telomeric repeats at the ade $^{+}$locus in the Type B clone, we found that the internal telomeric repeats became associated with the SPB in the horsetail stage (data not shown). Therefore, the presence of telomeric repeats at the physical DNA end is not required for establishing the telomere-SPB association. The highly concentrated local accumulation of Tazl recruited by tandem arrayed telomeric repeats may be critical for the establishment.

The circular chromosomes most likely resulted from the insufficient capping function of the original shortened telomeres in $\Delta$ trt 1 . Therefore, it is evident that the epigenetically inherited complex responsible for the subtelomere-SPB association does not possess all aspects of telomere function. This reasoning emphasizes the possibility that different levels of complexes responsible for the diverse functions of the telomere are maintained via different mechanisms, although some components are shared by the complexes. We observed that Swi6 was colocalized at TAS in Type B cells at a higher frequency than that expected from the poor Tazl colocalization (Fig. 5A). Moreover, Taz1 was dispensable for Swi6 localization in wild-type cells (Fig. 5B). Although Tazl and Swi6 play important roles in many aspects of telomere function (Ekwall et al. 1995; Cooper et al. 1997, 1998; Nimmo et al. 1998), the two proteins may be present in separable complexes in both mitotic and premeiotic horsetail stages.

\section{Materials and methods}

Yeast strains and plasmids

All strains were constructed from PM26-1 ( $h^{90}$ ade6-M210 leu132 ura4-D18). Derivative clones a-h (named SPTN242SPTN249) were obtained by successive plating of independent PM26-1 trt1::LEU2 transformants on YPD. Loss of telomeric repeats and chromosome circularization were confirmed by Southern hybridization and pulsed-field gel electrophoresis, respectively (data not shown). Derivative clones carrying Swi6GFP were constructed by integrating pTN138 (swi $\left.6^{+}-G F P\right)$ into the $s w i 6^{+}$locus, and were cultured in the presence of $0.5 \mu \mathrm{M}$ thiamine. In this clone, swi $6^{+}-G F P$ and untagged $s w i 6^{+}$were expressed from the native promoter and the $n m t 1$ promoter, respectively. $\Delta t a z 1$ strains were constructed by transformation with pAM24 (taz1::ura4 ${ }^{+}$) or pAM23 (taz1::LEU2). HA tagging of Taz1 was carried out as follows: pTN149 was constructed by deleting the XhoI-PstI fragment of pAMP157, a derivative of pSLF172 (Forsburg and Sherman 1997). The C-terminal $711 \mathrm{bp}$ of taz1 $^{+}$ORF was PCR-amplified, cloned into pTN149, and named pTN151. PM26-1, SPTN242, and SPTN243 were transformed with EcoRV-digested pTN151 to obtain SPTN327, SPTN329, and SPTN331, respectively. Taz1-HA reintroduction into $\Delta t a z 1$ clones was carried out by transformation with SnaBIdigested pYB169. Taz1-HA was expressed from the native promoter in this transformant. The expression of Tazl-HA was confirmed by immunoblotting with anti-HA antibodies (16B12; BabCO). Unpublished sequence information of pNSU56 was 
kindly provided by T.M. Nakamura (Department of Molecular Biology, The Scripps Research Institute, La Jolla, CA).

\section{Southern hybridization}

Genomic Southern hybridization was carried out as described (Sambrook et al. 1989). The locations of the probes are shown in Figure 2A. The TAS2 probe was cut out from pNSU70. The E11-E14 and 562 probes were prepared by PCR.

\section{Fluorescence in situ hybridization (FISH)} and immunostaining

FISH probes were prepared as follows. Plasmid or cosmid clones were prepared using a Plasmid Mega kit (QIAGEN). They were digested with AluI, Sau3AI, RsaI, HaeI, and DdeI, and then purified using Microcon 30 (Millipore). Digested DNA was labeled with Cy3-dCTP (Amersham Pharmacia) using a Random Primer DNA Labeling Kit (Takara). Unincorporated Cy3-dCTP was removed using Microcon 10 (Millipore). The labeled DNA was resuspended in TE to give $100 \mathrm{ng} / \mu \mathrm{L}$, and stored at $-20^{\circ} \mathrm{C}$ until use. FISH and immunostaining were carried out following the method of Shimanuki et al. (1997) with slight modifications. Conjugated cells were collected and resuspended in PEM (100 mM PIPES, 1 mM EGTA, $1 \mathrm{mM} \mathrm{MgSO}_{4}$ at $\mathrm{pH}$ 6.9) containing $3 \%$ paraformaldehyde and $0.2 \%$ glutaraldehyde, and then incubated with shaking at $26^{\circ} \mathrm{C}$ for $0.5-1 \mathrm{~h}$. Cells were collected and washed with PEM, incubated in PEM containing 0.1 M glycine, and then washed with PEMS (PEM with $1.2 \mathrm{M}$ sorbitol). Cells were suspended in PEMS containing $0.5 \mathrm{mg} / \mathrm{mL}$ Zymolyase 100T (Seikagakukogyo) and $0.1 \mathrm{mg} / \mathrm{mL}$ Novozyme 234 (Novo Nordisk), and incubated at $37^{\circ} \mathrm{C}$ for $10-30 \mathrm{~min}$ (for mitotic cells, incubated for 90-150 min). Cells were collected and washed with PEMS. Cells were suspended in PEMS containing $0.1 \%$ Triton X-100 and incubated for 2 min, followed by washing with PEMS and PEM. Cells were resuspended in PEMBAL (PEM with $1 \%$ BSA, $0.1 \%$ sodium azide, $1 \%$ lysine $\mathrm{HCl}$ ) containing primary antibody (for staining SPB, rabbit anti-Sad1 antibody was used) and incubated for 12-16 h. Cells were washed with PEMBAL, resuspended in PEMBAL containing fluorophore-conjugated secondary antibody (Molecular Probes), and incubated for 12-16 h. Cells were washed with PEMBAL, and antibodies were fixed with $3 \%$ formaldehyde for $20 \mathrm{~min}$, and then cells were washed with PEM. Then, the cells were resuspended in hybridization buffer containing $0.1 \mu \mathrm{g}$ of Cy3-labeled DNA probes, heated at $75^{\circ} \mathrm{C}$ for $5 \mathrm{~min}$, and hybridized at $40^{\circ} \mathrm{C}$ for 12-16 h. Cells were then washed with $2 \times$ SSC and resuspended in PBS containing $0.2 \mu \mathrm{g} / \mathrm{mL}$ DAPI. For staining of cells expressing Tazl-HA, fixation was done with $3 \%$ paraformaldehyde only, cell wall digestion was performed for $5 \mathrm{~min}$, and anti-HA antibody (16B12; BabCO) was used. Images were captured using an Axioskop (Zeiss) equipped with a CCD camera (Photometrics) operated by IP Lab Spectrum software (Signal Analytics), and were then processed with Photoshop 5.0 (Adobe).

\section{ChIP}

ChIP was carried out according to the method of Saitoh et al. (1997). Taz1-HA-expressing cells in $50 \mathrm{~mL}$ of YPD culture $\left(2 \times 10^{7} / \mathrm{mL}\right)$ were fixed with formaldehyde. For immunoprecipitation, anti-HA antibody (16B12; BabCO) and Protein A Sepharose CL-4B (Amersham Pharmacia) were used. Portions of immunoprecipitated DNAs were used as the PCR template. The locations of the primer sets are shown in Figure 3A.

\section{Southern hybridization-based ChIP}

The 562, 70TEL, E11, and $K$ region fragments were prepared by PCR and run on agarose gel in duplicate lanes. The gel was blotted onto a nylon membrane sheet. Immunoprecipitated DNAs from strains expressing Taz1-HA or untagged Tazl (DNA samples identical to those used in the PCR-based ChIP assayl were labeled using the Random Primed DNA Labeling Kit (Roche), and hybridized the membrane-blotted DNA.

\section{Acknowledgments}

We thank O. Niwa for FISH protocols and anti-Sad1 antibody, M. Yanagida for ChIP protocols, and T.M. Nakamura for pNSU56 sequences. We also thank S. Enomoto, S.I.S. Grewal, and our laboratory members for helpful discussion and support; as well as A. Nabetani and J. Kanoh for critical reading of the manuscript. J.-I. Nakayama is particularly acknowledged not only for supplying materials but also for his continuous encouragement and sincere discussion. This work was supported by a COE Grant; Grants-in-Aid for Cancer Research from the Ministry of Education, Culture, Sports, Science and Technology; and a Grant-in-Aid from the Virtual Research Institute of Aging (VRIA) of Nippon Boehringer Ingelheim. T.N. was supported by a JSPS Research Fellowship for Young Scientists.

The publication costs of this article were defrayed in part by payment of page charges. This article must therefore be hereby marked "advertisement" in accordance with 18 USC section 1734 solely to indicate this fact.

\section{References}

Allshire, R.C., Javerzat, J.P., Redhead, N.J., and Cranston, G. 1994. Position effect variegation at fission yeast centromeres. Cell 76: 157-169.

Allshire, R.C., Nimmo, E.R., Ekwall, K., Javerzat, J.P., and Cranston, G. 1995. Mutations derepressing silent centromeric domains in fission yeast disrupt chromosome segregation. Genes \& Dev. 9: 218-233.

Barnett, M.A., Buckle, V.J., Evans, E.P., Porter, A.C., Rout, D., Smith, A.G., and Brown, W.R. 1993. Telomere directed fragmentation of mammalian chromosomes. Nucleic Acids Res. 21: 27-36.

Chikashige, Y. and Hiraoka, Y. 2001. Telomere binding of the Rap1 protein is required for meiosis in fission yeast. Curr. Biol. 11: 1618-1623.

Chikashige, Y., Ding, D.Q., Funabiki, H., Haraguchi, T., Mashiko, S., Yanagida, M., and Hiraoka, Y. 1994. Telomereled premeiotic chromosome movement in fission yeast. Science 264: 270-273.

Cooper, J.P. 2000. Telomere transitions in yeast: The end of the chromosome as we know it. Curr. Opin. Genet. Dev. 10: $169-177$.

Cooper, J.P., Nimmo, E.R., Allshire, R.C., and Cech, T.R. 1997. Regulation of telomere length and function by a Myb-domain protein in fission yeast. Nature 385: 744-747.

Cooper, J.P., Watanabe, Y., and Nurse, P. 1998. Fission yeast Tazl protein is required for meiotic telomere clustering and recombination. Nature 392: 828-831.

Ekwall, K., Javerzat, J.P., Lorentz, A., Schmidt, H., Cranston, G., and Allshire, R. 1995. The chromodomain protein Swi6: A key component at fission yeast centromeres. Science 269: 1429-1431.

Ekwall, K., Olsson, T., Turner, B.M., Cranston, G., and Allshire, R.C. 1997. Transient inhibition of histone deacetylation alters the structural and functional imprint at fission yeast centromeres. Cell 91: 1021-1032. 
Forsburg, S.L. and Sherman, D.A. 1997. General purpose tagging vectors for fission yeast. Gene 191: 191-195.

Funabiki, H., Hagan, I., Uzawa, S., and Yanagida, M. 1993. Cell cycle-dependent specific positioning and clustering of centromeres and telomeres in fission yeast. J. Cell Biol. 121: 961-976.

Gottschling, D.E., Aparicio, O.M., Billington, B.L., and Zakian, V.A. 1990. Position effect at $S$. cerevisiae telomeres: Reversible repression of Pol II transcription. Cell 63: 751-762.

Grewal, S.I. and Klar, A.J. 1996. Chromosomal inheritance of epigenetic states in fission yeast during mitosis and meiosis. Cell 86: 95-101.

- 1997. A recombinationally repressed region between mat2 and mat 3 loci shares homology to centromeric repeats and regulates directionality of mating-type switching in fission yeast. Genetics 146: 1221-1238.

Hagan, I. and Yanagida, M. 1995. The product of the spindle formation gene $\mathrm{sad1}^{+}$associates with the fission yeast spindle pole body and is essential for viability. J. Cell Biol. 129: $1033-1047$.

Hall, I.M., Shankaranarayana, G.D., Noma, K., Ayoub, N., Cohen, A., and Grewal, S.I. 2002. Establishment and maintenance of a heterochromatin domain. Science 297:22322237.

Hiraoka, Y. 1998. Meiotic telomeres: A matchmaker for homologous chromosomes. Genes Cells 3: 405-413.

Ishikawa, F. and Naito, T. 1999. Why do we have linear chromosomes? A matter of Adam and Eve. Mutat. Res. 434: 99107.

Kanoh, J. and Ishikawa, F. 2001. spRap1 and spRif1, recruited to telomeres by Taz1, are essential for telomere function in fission yeast. Curr. Biol. 11: 1624-1630.

Karpen, G.H. and Allshire, R.C. 1997. The case for epigenetic effects on centromere identity and function. Trends Genet. 13: 489-496.

Lieb, J.D., Liu, X., Botstein, D., and Brown, P.O. 2001. Promoterspecific binding of Rap1 revealed by genome-wide maps of protein-DNA association. Nat. Genet. 28: 327-334.

Lundblad, V. and Blackburn, E.H. 1993. An alternative pathway for yeast telomere maintenance rescues est $1^{-}$senescence. Cell 73: 347-360.

Mefford, H.C. and Trask, B.J. 2002. The complex structure and dynamic evolution of human subtelomeres. Nat. Rev. Genet. 3: 91-102.

Naito, T., Matsuura, A., and Ishikawa, F. 1998. Circular chromosome formation in a fission yeast mutant defective in two ATM homologues. Nat. Genet. 20: 203-206.

Nakamura, T.M., Cooper, J.P., and Cech, T.R. 1998. Two modes of survival of fission yeast without telomerase. Science 282: 493-496.

Nakayama, J., Klar, A.J., and Grewal, S.I. 2000. A chromodomain protein, Swi6, performs imprinting functions in fission yeast during mitosis and meiosis. Cell 101: 307-317.

Nimmo, E.R., Cranston, G., and Allshire, R.C. 1994. Telomereassociated chromosome breakage in fission yeast results in variegated expression of adjacent genes. EMBO J. 13: 38013811.

Nimmo, E.R., Pidoux, A.L., Perry, P.E., and Allshire, R.C. 1998. Defective meiosis in telomere-silencing mutants of Schizosaccharomyces pombe. Nature 392: 825-828.

Niwa, O., Shimanuki, M., and Miki, F. 2000. Telomere-led bouquet formation facilitates homologous chromosome pairing and restricts ectopic interaction in fission yeast meiosis. EMBO J. 19: 3831-3840.

Saitoh, S., Takahashi, K., and Yanagida, M. 1997. Mis6, a fission yeast inner centromere protein, acts during G1/S and forms specialized chromatin required for equal segregation. Cell 90: 131-143.

Sambrook, J., Fritsch, E.F., and Maniatis, T. 1989. Molecular cloning: A laboratory manual, 2nd ed. Cold Spring Harbor Laboratory Press, Cold Spring Harbor, NY.

Sandell, L.L. and Zakian, V.A. 1993. Loss of a yeast telomere: Arrest, recovery, and chromosome loss. Cell 75: 729-739.

Scherthan, H. 2001. A bouquet makes ends meet. Nat. Rev. Mol. Cell. Biol. 2: 621-627.

Scherthan, H., Bahler, J., and Kohli, J. 1994. Dynamics of chromosome organization and pairing during meiotic prophase in fission yeast. J. Cell Biol. 127: 273-285.

Shimanuki, M., Miki, F., Ding, D.Q., Chikashige, Y., Hiraoka, Y., Horio, T., and Niwa, O. 1997. A novel fission yeast gene, $\mathrm{kms} 1^{+}$, is required for the formation of meiotic prophasespecific nuclear architecture. Mol. Gen. Genet. 254: 238249.

Smith, C.D., Smith, D.L., DeRisi, J.L., and Blackburn, E.H. 2003. Telomeric protein distributions and remodeling through the cell cycle in Saccharomyces cerevisiae. Mol. Biol. Cell. 14: 556-570.

Spink, K.G., Evans, R.J., and Chambers, A. 2000. Sequence-specific binding of Tazlp dimers to fission yeast telomeric DNA. Nucleic Acids Res. 28: 527-533.

Strahl-Bolsinger, S., Hecht, A., Luo, K., and Grunstein, M. 1997. SIR2 and SIR4 interactions differ in core and extended telomeric heterochromatin in yeast. Genes \& Dev. 11: 83-93.

Sugawara, N. 1989. "DNA sequences at the telomeres of the fission yeast $S$. pombe." Ph.D thesis. Harvard University.

Sullivan, B.A., Blower, M.D., and Karpen, G.H. 2001. Determining centromere identity: Cyclical stories and forking paths. Nat. Rev. Genet. 2: 584-596.

Varley, H., Di, S., Scherer, S.W., and Royle, N.J. 2000. Characterization of terminal deletions at $7 \mathrm{q} 32$ and $22 \mathrm{q} 13.3$ healed by de novo telomere addition. Am. J. Hum. Genet. 67: 610 622.

Vassetzky, N.S., Gaden, F., Brun, C., Gasser, S.M., and Gilson, E. 1999. Tazlp and Teblp, two telobox proteins in Schizosaccharomyces pombe, recognize different telomere-related DNA sequences. Nucleic Acids Res. 27: 4687-4694.

Wang, G., Ma, A., Chow, C.M., Horsley, D., Brown, N.R., Cowell, I.G., and Singh, P.B. 2000. Conservation of heterochromatin protein 1 function. Mol. Cell. Biol. 20: 6970-6983.

Wood, V., Gwilliam, R., Rajandream, M.A., Lyne, M., Lyne, R., Stewart, A., Sgouros, J., Peat, N., Hayles, J., Baker, S., et al. 2002. The genome sequence of Schizosaccharomyces pombe. Nature 415: 871-880. 


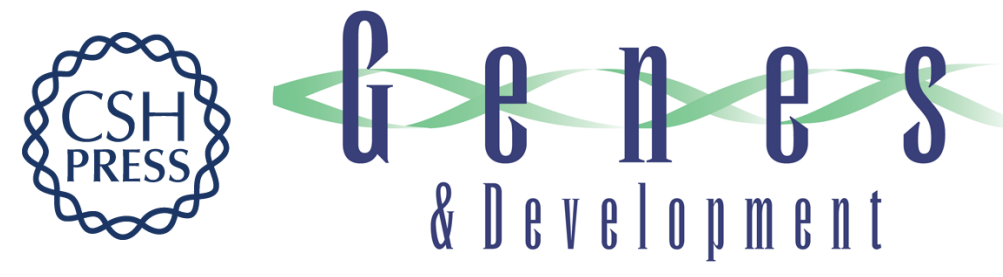

\section{Stable inheritance of telomere chromatin structure and function in the absence of telomeric repeats}

Mahito Sadaie, Taku Naito and Fuyuki Ishikawa

Genes Dev. 2003, 17:

Access the most recent version at doi:10.1101/gad.1112103

Supplemental http://genesdev.cshlp.org/content/suppl/2003/09/03/1112103.DC1
Material

References This article cites 43 articles, 13 of which can be accessed free at: http://genesdev.cshlp.org/content/17/18/2271.full.html\#ref-list-1

License

Email Alerting Receive free email alerts when new articles cite this article - sign up in the box at the top Service right corner of the article or click here.

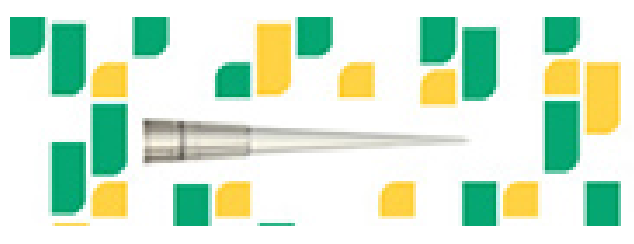

Focused on your science. 